\title{
Optical manipulation of quantum dot excitons strongly coupled to photonic crystal cavities
}

\author{
Arka Majumdar ${ }^{* 1}$, Andrei Faraon ${ }^{2}$, Dirk Englund ${ }^{3}$, Nicolas Manquest ${ }^{1}$, Hyochul Kim ${ }^{4}$, \\ Pierre Petroff 4 ,Jelena Vučković ${ }^{1}$ \\ ${ }^{1}$ E.L. Ginzton Laboratory, Stanford University, Stanford, CA-94305 \\ ${ }^{2}$ H.P. Laboratories, Palo Alto, CA \\ ${ }^{3}$ Columbia University, New York City, NY \\ 4 Materials Department, University of California, Santa Barbara, CA
}

\begin{abstract}
In this paper, we review some recent cavity quantum electrodynamic (CQED) experiments with single quantum dot exciton coupled to photonic crystal cavities, performed in our group. We show how the coupled quantumdot/cavity system can be used to modulate light with at a very fundamental level with very low power and discuss some applications of these low power modulators.
\end{abstract}

Keywords: quantum information, photonic crystal, quantum dot, electro-optic modulation, all-optical modulation

\section{INTRODUCTION}

Integration of single quantum dot (QD) in photonic crystal cavity enabled quantum optical experiments in solid state over the past few years. ${ }^{1-4}$ This system can be used to design robust, scalable quantum information processing devices. ${ }^{1}$ In addition this coupled system is a promising candidate for classical optical information processing. In fact, the fidelity required in quantum information processing is much more stringent compared to the fidelity needed for classical information processing, and hence it is likely that this system will find use in classical information processing much before it is used in quantum information systems.

Photonic crystal (PC) is a periodic array of materials with different refractive indices. This periodicity gives rise to a photonic band-gap. If a defect is introduced in this periodicity, the light remains confined in-plane by distributed Bragg reflection (DBR) and out-of-plane by total internal reflection. Fig. 1 shows some scanning electron micro-graph of photonic crystal integrated structures.

On the other hand in a quantum dot (QD), electrons and holes are confined in three dimensions. This type of three-dimensional confinement gives rise to delta function like density of states and QD can be treated like an artificial atom. In our experiments, the ground state to the single exciton state transition is used as the atomic transition. Thus a single QD coupled to cavity enables study of light-matter interaction at the most fundamental level, where single photon and single exciton can significantly affect the system performance.

In this paper, we review several recent experiments with coupled QD-cavity system, done by our group. In section 2, the physics of a coupled QD-cavity system is explained. Section 3 explains how this system can be used for all-optical switching. Section 4 explains the effect of external electric field on the QD and possible use of the coupled system in electro-optic modulator. In section 5, we describe a novel scheme of optical modulation, where both optical and electrical effects are exploited.

\footnotetext{
*To whom correspondence should be addressed. Email: arkam@stanford.edu
} 
a

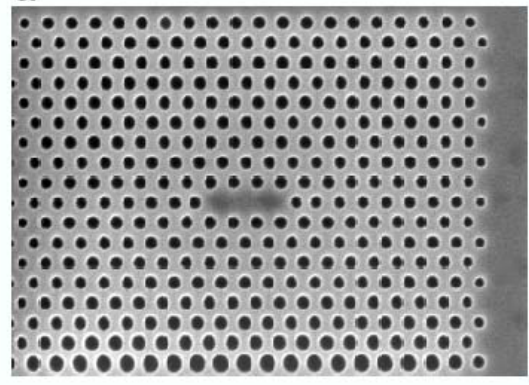

b
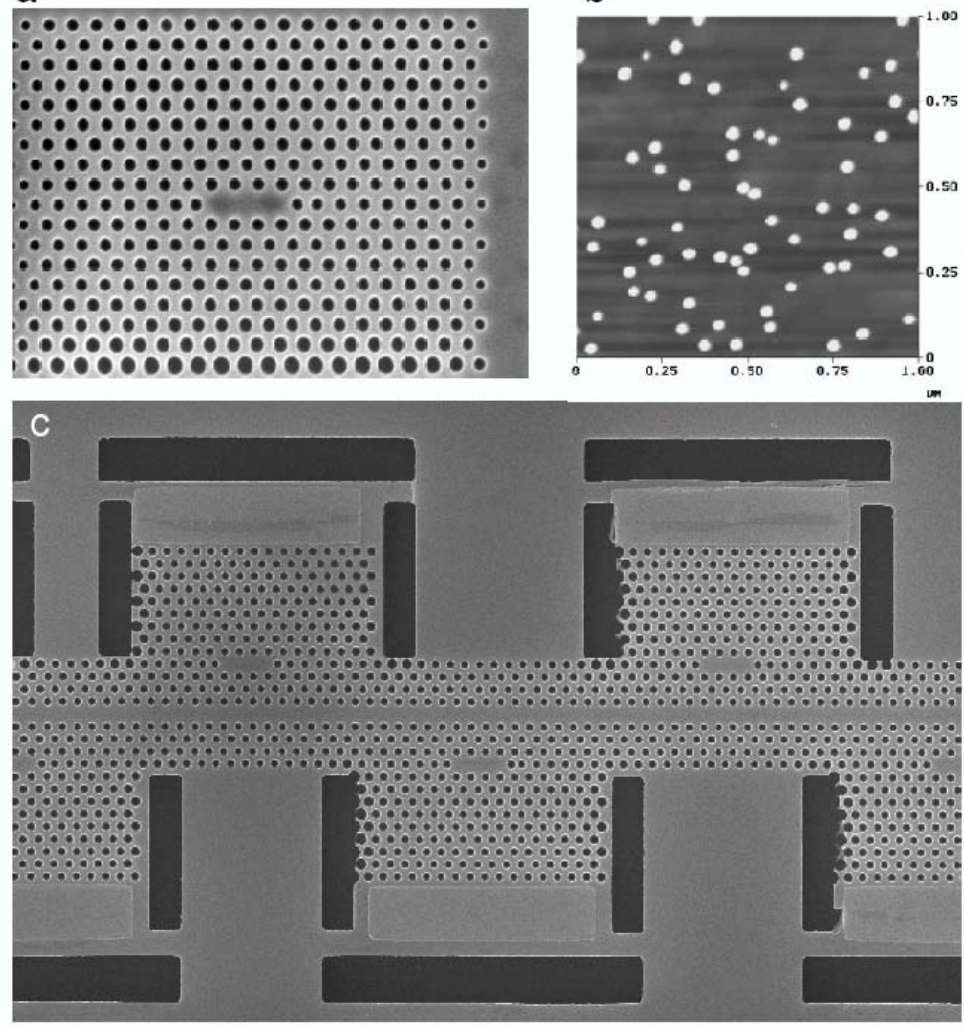

Figure 1. (a)Scanning electron microscope image of the fabricated structure showing the photonic crystal structure and the cavity, (b) A chip containing Quantum Dots, (c) Scanning electron micro-graph of a hypothetical integrated nano-photonic structure made of photonic crystal cavities and wave-gudies.

\section{STRONG COUPLING}

The dynamics of a driven coupled QD-cavity system is described by the Master equation ${ }^{5}$

$$
\frac{d \rho}{d t}=-\frac{i}{\hbar}\left[H_{J C}+H_{d}, \rho\right]+\kappa \mathcal{L}[a]+\gamma \mathcal{L}[\sigma]
$$

Here, $\rho$ is the density matrix of the coupled QD-cavity system, $\kappa$ is the cavity decay rate and $\gamma$ is the QD spontaneous emission rate. $\mathcal{L}[D]$ is the Lindbald operator for an operator $D$ and is given by

$$
\mathcal{L}[D]=D \rho D^{\dagger}-\frac{1}{2} D^{\dagger} D \rho-\frac{1}{2} \rho D^{\dagger} D
$$

$H_{J C}$ is Jaynes-Cummings Hamiltonian describing the coherent interaction between the QD and the cavity and is given by

$$
H_{J C}=\hbar \omega_{c} a^{\dagger} a+\hbar \omega_{a} \sigma^{\dagger} \sigma+\hbar g\left(\sigma^{\dagger} a+\sigma a^{\dagger}\right)
$$

and $H_{d}$ signifies the coherent driving of the cavity mode and is given by

$$
H_{d}=E\left(a e^{-i \omega_{l} t}+a^{\dagger} e^{-i \omega_{l} t}\right)
$$

Here, $\omega_{c}$ and $\omega_{a}$ are the cavity and the QD resonance frequency respectively, $\omega_{l}$ is the driving laser frequency, $\sigma$ is the lowering operator for the QD, $a$ is the annihilation operator for the cavity photon; $g$ is the coherent interaction strength between the $\mathrm{QD}$ and the cavity and $E$ denotes the driving strength of the laser field. The total Hamiltonian $H=H_{J C}+H_{d}$ under rotating wave approximation (RWA) is given by

$$
H=\hbar \Delta \omega_{c} a^{\dagger} a+\hbar \Delta \omega_{a} \sigma^{\dagger} \sigma+\hbar g\left(\sigma^{\dagger} a+\sigma a^{\dagger}\right)+E\left(a+a^{\dagger}\right)
$$




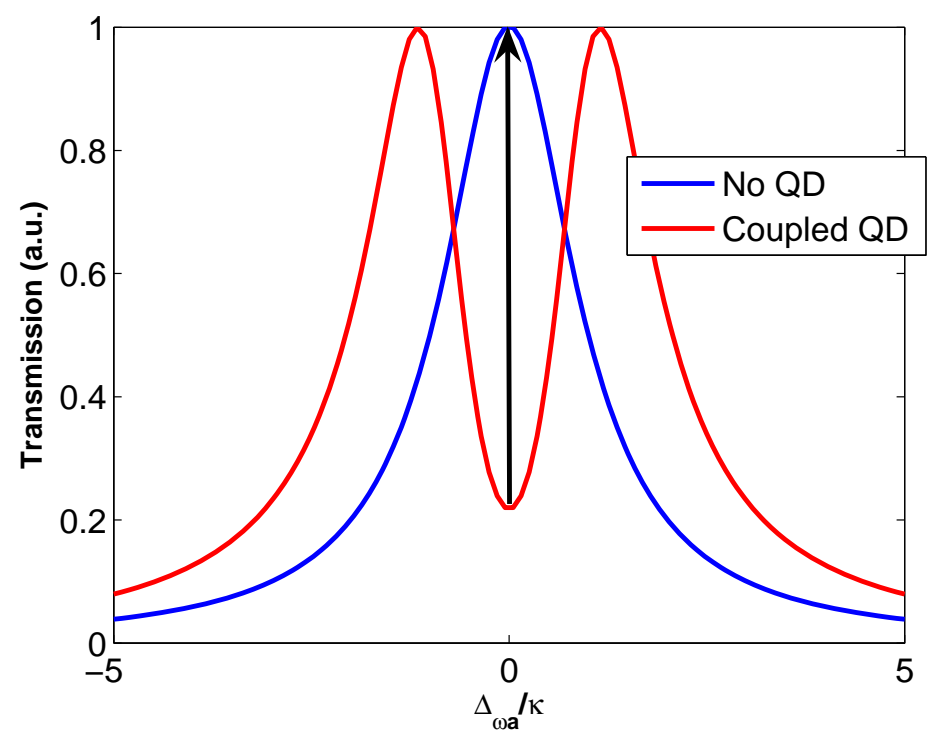

Figure 2. Calculated transmission spectra of an empty cavity and a strongly coupled QD-cavity system. For the simulation, $g / 2 \pi=\kappa / 2 \pi=20 \mathrm{GHz}$ is assumed. The black arrow shows the change in transmission of the laser resonant to the empty cavity frequency.

where, $\Delta \omega_{c}=\omega_{c}-\omega_{l}$ and $\Delta \omega_{a}=\omega_{a}-\omega_{l}$ are the detuning of the laser from the cavity and the QD respectively.

When the coherent interaction strength $g$ is much greater than the losses in the system, i.e., the cavity decay rate $\kappa$ and the QD spontaneous emission rate $\gamma$, the system is in strong coupling regime. In strong coupling regime, the eigen-states of $H_{J C}$ are polaritons, possessing characteristics of both the cavity and the QD. Fig. 2 shows the calculated transmission spectra of the coupled QD-cavity system, when a QD is strongly coupled and when it is not. Hence the transmission of a laser that is resonant with the empty cavity frequency can be changed by the presence of the QD. Transmission of the laser is maximum when the laser is resonant with the cavity and no QD is coupled. However, when a coupled QD is present, the light scattered by the QD and the incoming light destructively interfere ${ }^{6}$ and light transmission is significantly reduced. This phenomenon is called dipole induced transparency (DIT). Hence by modifying the QD resonance, we can modulate the laser transmission through the cavity.

\section{ALL OPTICAL SWITCHING}

As explained above, we can control the light transmission by controlling the QD. One way to control the QD, is by optically saturating the dot. A strongly driven QD saturates when the rate of QD excitation becomes same as QD spontaneous emission rate. In that case, the empty cavity spectrum is recovered. The calculated transmission of the laser through a coupled QD-PC cavity system as a function of driving laser power is shown in Fig. 3. To calculate the speed of optical modulation via saturation of the QD we simulated a coupled QDcavity system with pulsed control and signal inputs. The time dependent Hamiltonian under RWA is given by (assuming the driving laser is resonant to both the cavity and the QD)

$$
H=\hbar g\left(\sigma^{\dagger} a+\sigma a^{\dagger}\right)+\Omega_{c}(t)\left(a+a^{\dagger}\right)+\Omega_{s}(t)\left(a+a^{\dagger}\right)
$$

Here, $\Omega_{c}(t)$ and $\Omega_{s}(t)$ are the time dependent driving term for control and signal respectively. Fig. 4 shows the theoretical estimate of the time integrated cavity output $\left\langle a^{\dagger}(t) a(t)\right\rangle$ as a function of the delay between the control and signal pulses, for different power of the control and signal. We observe that the cavity output shows a peak at around zero delay. This shows that when two pulses come together, they saturate the QD and we get more laser transmission. This optical nonlinearity happens in single photon-single QD regime and provides non-linear light-matter interaction at the most fundamental level. Fig. 5 shows the experimental data of cavity transmission as a function of the delay between two pulses. Streak camera is used to perform the experiment. 


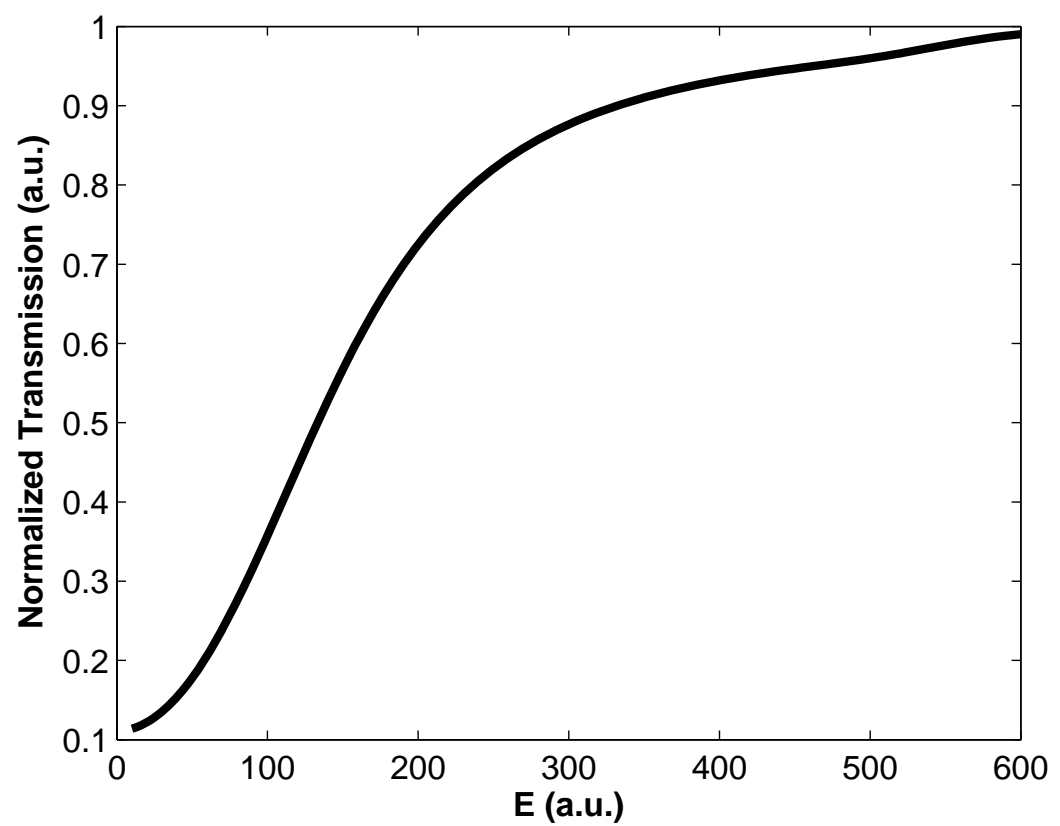

Figure 3. Calculated normalized transmission of a laser through the coupled QD-cavity system as a function driving field amplitude. The system parameters are $g / 2 \pi=\kappa / 2 \pi=20 \mathrm{GHz} ; \gamma / 2 \pi=1 \mathrm{GHz}$. The transmission through the coupled system is normalized by the transmission through an empty cavity.

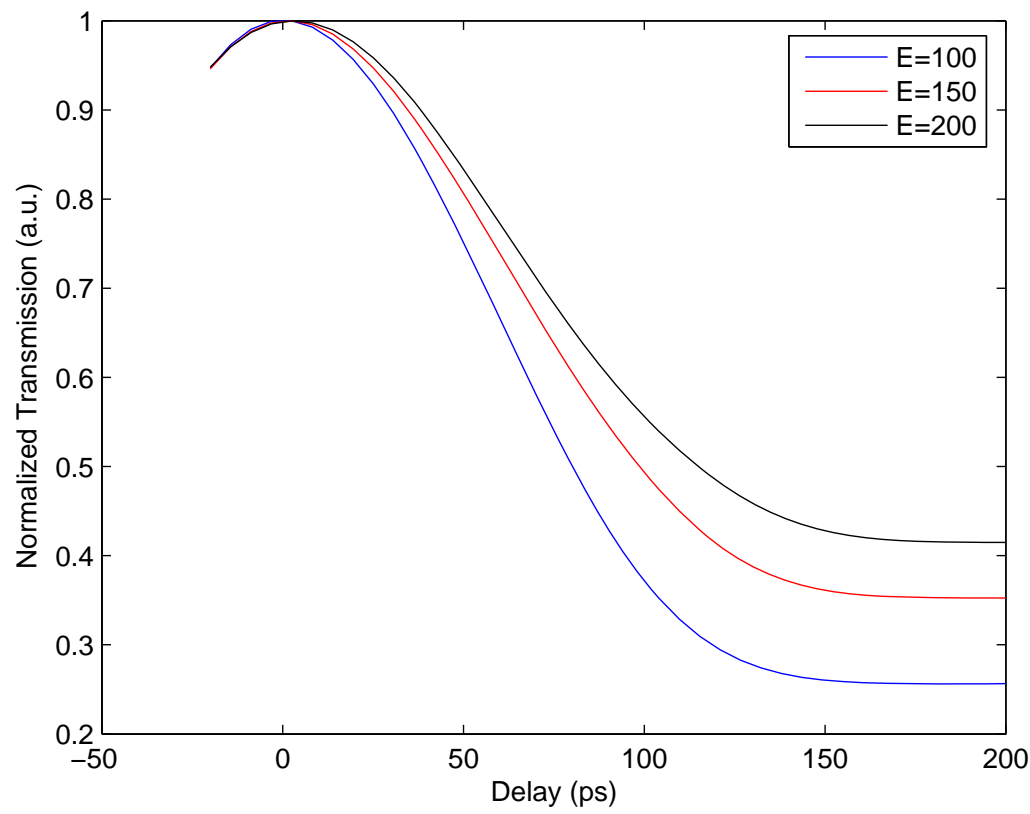

Figure 4. Calculated time integrated cavity transmission through a coupled QD-cavity system as a function of the delay between two pulses, for different values of $E$. The system parameters are $g / 2 \pi=\kappa / 2 \pi=20 \mathrm{GHz} ; \gamma / 2 \pi=1 \mathrm{GHz}$. Control and signal are assumed to have same intensity. The transmission is normalized by the maximum transmission which happens at zero time delay. 


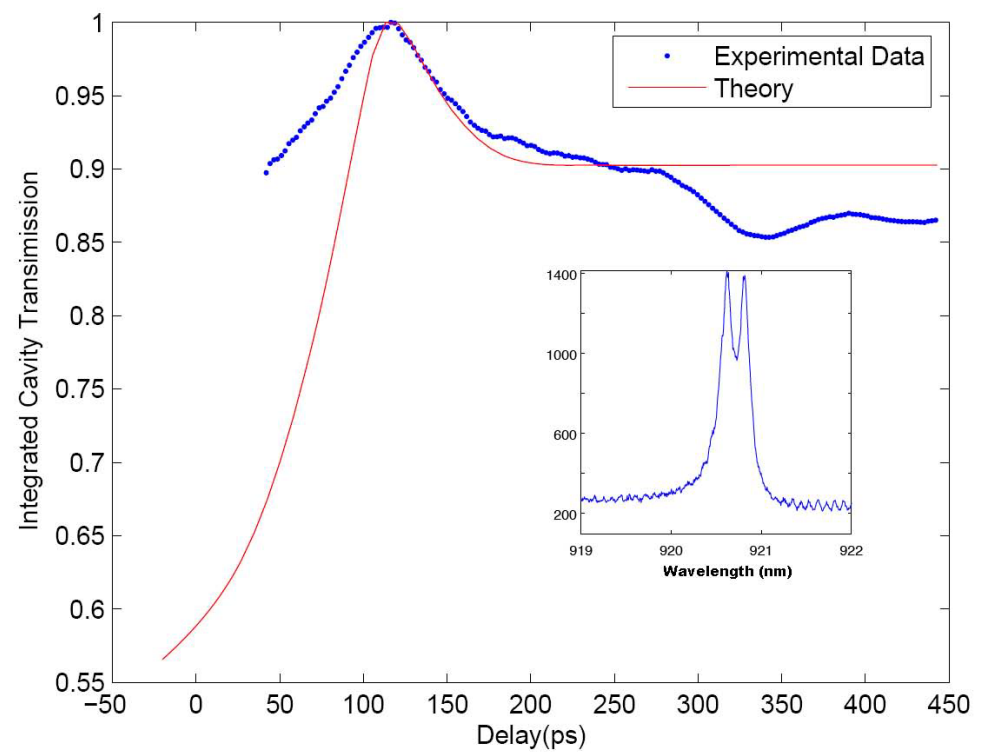

Figure 5. Time integrated cavity transmission through a coupled QD-cavity system as a function of the delay between two pulses. The solid red line is obtained via simulation. Pulses used for the experiment are 40 ps long. (Inset) Reflectivity spectrum of the experimental system. For the experimental system $g / 2 \pi=25 \mathrm{GHz} \kappa / 2 \pi=40 \mathrm{GHz}$.

\section{ELECTRO OPTIC SWITCHING}

Increasing metallic losses at high frequency in modern electronic interconnects pose a serious problem. Optical interconnects are considered a viable solution. To integrate photonics with electronics, one needs to convert electronic signal to optical signal. One way to achieve this is to use an electro-optical modulator. However, for all existing electro-optic modulators the control power requirement is very high. ${ }^{7}$ A single QD coupled to cavity can modulate the optical signal with a very low control power as the active volume of the system is very small.

The principle behind the electro-optic modulation is: the QD resonance is controlled by electrical signal via quantum confined Stark effect (QCSE). Modulation of QD resonance control the laser transmission through the cavity. We use a lateral electric field to shift the QD resonance ${ }^{8}$ and thus modulate the light. Fig. 6 shows the modulation of light at a frequency of $150 \mathrm{MHz}$, limited by the $\mathrm{RC}$ constant of the transmission line. However, the control power for modulation is very small $(\sim 1)$ fJ. The modulator can be thought of as a capacitor, that needs to be charged. As the active volume of the QD is very small $10 \times 10 \times 10 \mathrm{~nm}^{3}$, the capacitance is very small and so is the control power.

Though experimentally achieved modulation frequency is around $150 \mathrm{MHz}$, theoretically this modulator can provide modulation frequency of $10-20 \mathrm{GHz}$. In experiment, the modulation speed is limited by the imperfect termination of the BNC cable carrying signal to the chip. The Hamiltonian describing the dynamics of the modulated system is given by

$$
H=\Delta \omega_{c} a^{\dagger} a+\Delta \omega_{a}(t) \sigma^{\dagger} \sigma+i g\left(a^{\dagger} \sigma-a \sigma^{\dagger}\right)+\Omega\left(a+a^{\dagger}\right)
$$

where, $\Delta \omega_{a}(t)$ is the effect of modulation of QD frequency. We assume a sinusoidal modulation of the form $\Delta \omega_{a}(t)=\frac{1}{2} \Delta \omega_{0}\left(1-\cos \left(\omega_{e} t\right)\right)$, where, where $\Delta \omega_{0}$ is the maximum detuning of the QD resonance (this is proportional to the amplitude of the electrical signal applied to tune the QD) and $\omega_{e}$ is the frequency of the modulating electrical signal and hence also the frequency of the change in the QD resonance. The output is given by the expected value of $\left\langle a^{\dagger} a\right\rangle$. We change the modulation frequency and observe the on/off ratio of the modulator. The filter response of this modulator is shown in Fig.7 for different cavity decay rate $\kappa$ and coherent coupling strength $g .^{9}$ 

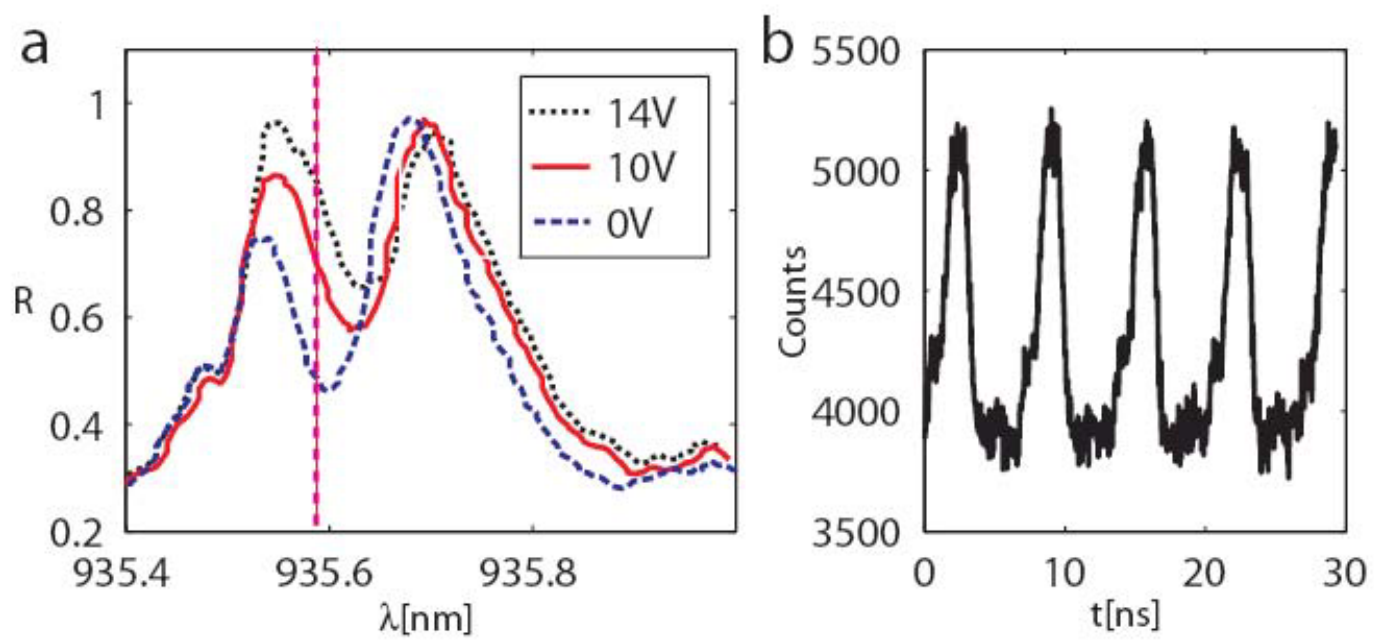

Figure 6. (a) Experimentally obtained cavity transmission spectra for three different voltage. The QD resonance is affected by QCSE. The dotted line shows the laser wavelength, whose transmission is to be modulated. (b) The modulation of the laser with time. The modulation speed is $150 \mathrm{MHz}$. The time domain measurement is performed with a single photon counter and a pico-second time analyzer.

(a)

(b)
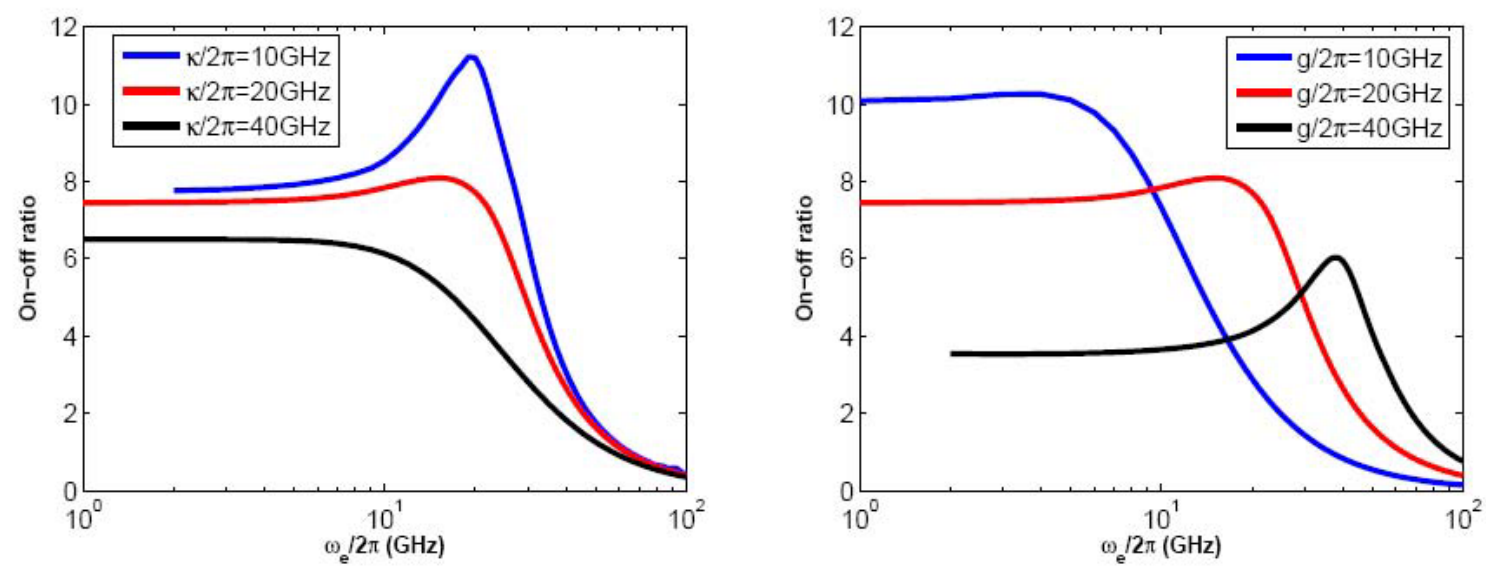

Figure 7. On-off ratio of the modulator based on a single QD strongly coupled to PC Cavity, as a function of the frequency of the electrical signal for different $g$ and $\kappa$. The modulator behaves as a first order low pass filter. 

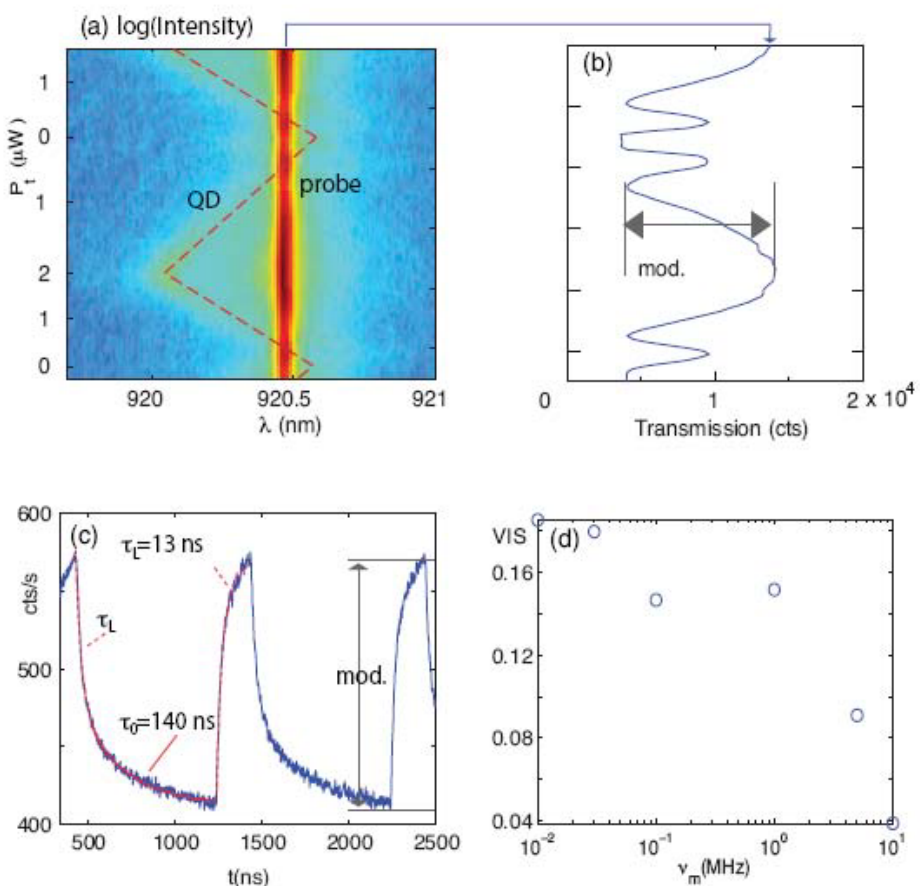

Figure 8. Modulating the cavity transmission by DC Stark shift of the QD. (a) The cavity transmission is probed by a narrow laser on resonance with the cavity as the dot is tuned across the cavity resonance. Pump laser power is $4 \mu \mathrm{W} ; P_{t}$ is varied between 0 and $2 \mu \mathrm{W}$; the probe laser power is $2 \mathrm{nW}$. (b) Transmission corresponding to (a) as the QD is tuned through the cavity. (c) Detector count rate I from the cavity transmission at a modulation frequency of $\nu_{m}=1 \mathrm{MHz}$. A double-exponential fit gives the response times $\tau_{L}=13 \mathrm{~ns}$ and $\tau_{0}=140 \mathrm{~ns}$. (d) Visibility (VIS) of the transmission as the modulation frequency $\nu_{m}$ is increased.

\section{ALL OPTICAL SWITCHING VIA ELECTRO-OPTIC EFFECT}

Apart from optical and electrical modulation of the optical signal, we propose another novel way to modulate the light, where both optical and electrical controls are involved. If the QD is placed in the intrinsic region of a p-i-n diode, the QD is affected by the built-in electric field of the diode. However, the effect of the electric field can be reduced by optically creating carriers and thus by screening the electric field. Change in electric field causes QCSE of the QD and this modulates the laser transmission.

Using this principle,we obtain modulation in transmission of laser through cavity. The modulation power needed is low, but the modulation speed is small $(\approx 1) \mathrm{MHz}$. We believe the lower speed is due to the imperfect growth of the sample. The charges are trapped in the defects and hence the decay of charges is slow. The experimental data ${ }^{10}$ is shown in Fig. 8. However by more careful fabrication one can increase the speed of the modulation.

\section{CONCLUSION}

In conclusion, we showed that coupled QD-cavity system can be used to tailor light-matter interaction at a very fundamental level. This enables e.g., very low power optical modulation. Cavity also enhances the efficiency to read out the information about the QD states, which can be used for future quantum information processing devices.

\section{ACKNOWLEDGEMENT}

The authors gratefully acknowledge financial support provided by the National Science Foundation, and Office of Naval Research. A.M. was supported by the Stanford Graduate Fellowship (Texas Instruments fellowship). 


\section{REFERENCES}

1. D. Englund, Andrei Faraon, Ilya Fushman, Nick Stoltz, Pierre Petroff, and Jelena Vuckovic. Controlling cavity reflectivity with a single quantum dot. Nature, 450:857, 2007.

2. K. Hennessy, A. Badolato, M. Winger, D. Gerace, M. Atature, S. Gulde, S. Falt, E. L. Hu, and A. Imamoglu. Quantum nature of a strongly-coupled single quantum dot-cavity system. Nature, 445:896, 2007.

3. K. Srinivasan and Oskar Painter. Linear and nonlinear optical spectroscopy of a strongly-coupled microdiskquantum dot system. Nature, 450:862, 2007.

4. Andrei Faraon, Ilya Fushman, Dirk Englund, Nick Stoltz, Pierre Petroff, and Jelena Vuckovic. Coherent generation of nonclassical light on a chip via photon-induced tunneling and blockade. Nature Physics, 450:859, 2008.

5. C. W. Gardiner and P. Zoller. Quantum Noise. Springer-Verlag, 2005.

6. Edo Waks and Jelena Vuckovic. Dipole induced transparency in drop-filter cavity-waveguide systems. Phys. Rev. Lett., 96(15):153601, Apr 2006.

7. D. A. B. Miller. Device requirements for optical interconnects to silicon chips. Proc. IEEE, 97:1166, 2009.

8. Andrei Faraon, Arka Majumdar, Hyochul Kim, Pierre Petroff, and Jelena Vuckovic. Fast electrical control of a quantum dot strongly coupled to a nano-resonator. arXiv:0906.0751v1, 2009.

9. Arka Majumdar, Nicolas Manquest, Andrei Faraon, and Jelena Vuckovic. Theory of electro-optic modulation via a quantum dot coupled to a nano-resonator. arXiv:0911.5207, 2009.

10. Dirk Englund, Andrei Faraon, Arka Majumdar, Nick Stoltz, Pierre Petroff, and Jelena Vuckovic. An optical modulator based on a single strongly coupled quantum dot - cavity system in a p-i-n junction. Optics Express, 17:18651, 2009. 